\section{Ultrasonographic features of traumatic neuromas in breast cancer patients after mastectomy}

\author{
Hwa Sung Sung, Young-Seon Kim \\ Department of Radiology, Yeungnam University College of Medicine, Daegu, Korea
}

Purpose: The purpose of this study was to evaluate the ultrasonographic (US) features of traumatic neuromas in breast cancer patients after mastectomy.

Methods: This study was performed with approval from our Institutional Review Board, and the requirement for informed consent was waived. Six traumatic neuromas in five patients were included in this study. The US findings of traumatic neuromas were evaluated retrospectively by two radiologists according to the Breast Imaging-Reporting and Data System (BI-RADS) lexicon. The final assessment was also recorded.

Results: On US, all six lesions presented as a mass within the pectoralis muscle layer (mean size, $4.8 \mathrm{~mm}$; range, 3.9 to $5.5 \mathrm{~mm}$ ). Of the six masses, four had an oval shape with a circumscribed margin, and two had an irregular shape and an indistinct margin. They were all hypoechoic. Two lesions showed a non-parallel orientation. On color Doppler examinations, two lesions showed internal vascularity. Strain elastography was performed for four neuromas, resulting in scores of $1(n=1), 4(n=2)$, and $5(n=1)$. The final assessment categories were BI-RADS $3(n=2), 4 A(n=2)$, and $4 B(n=2)$.

Conclusion: On US, an oval shape, circumscribed margin, parallel orientation, and hypoechogenicity were the most frequent features of traumatic neuromas in breast cancer patients after mastectomy. Neuromas may show increased vascularity on color Doppler imaging and present as a hard mass on elastography.

Keywords: Neuroma; Breast neoplasms; Mastectomy

\section{Introduction}

Traumatic neuromas are rare benign lesions that occur at the proximal end of a severed nerve [1]. They are not true neoplasms but hyperplastic proliferations of neuronal and connective tissue that occur in response to nerve injury [1]. They can occur in any part of the body that has been subjected to trauma or surgery, but most commonly occur in the lower extremities after amputation procedures, followed by the head and neck [2]. However, traumatic neuromas occurring in the breast or chest wall after surgery have been very rarely reported [2-10]. If a traumatic neuroma occurs in the breast or chest wall after oncologic surgery (breast-conserving surgery or mastectomy), it is difficult to clinically and radiologically differentiate from recurrence of the tumor, and a core needle biopsy or an excisional

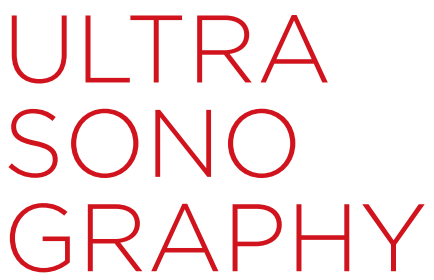

ORIGINAL ARTICLE

https://doi.org/10.14366/usg.16029 pISSN: 2288-5919 - elSSN: 2288-5943 Ultrasonography 2017;36:33-38

Received: June 8, 2016

Revised: July 13, 2016

Accepted: July 18, 2016

Correspondence to:

Young-Seon Kim, MD, Department of Radiology, Yeungnam University College of Medicine, 170 Hyeonchungro, Nam-gu, Daegu 42415, Korea

Tel. +82-53-620-4129

Fax. +82-53-653-5484

E-mail: ing29@hanmail.net

This is an Open Access article distributed under the terms of the Creative Commons Attribution NonCommercial License (http://creativecommons.org/ licenses/by-nc/3.0/) which permits unrestricted noncommercial use, distribution, and reproduction in any medium, provided the original work is properly cited.

Copyright (C) 2017 Korean Society of Ultrasound in Medicine (KSUM)

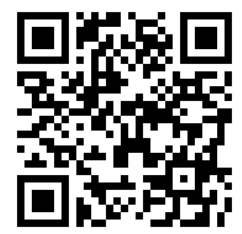

How to cite this article:

Sung HS, Kim YS. Ultrasonographic features of traumatic neuromas in breast cancer patients after mastectomy. Ultrasonography. 2017 Jan;36(1):33-38. 
biopsy must be performed to obtain a pathologic diagnosis. To our knowledge, the imaging findings of traumatic neuroma in the breast or chest wall after surgery have only been described in 21 cases: 20 cases in six reports written in English [2,4,6,8-10], and one case report involving a Korean patient [5]. The majority of the articles were individual case reports. The purpose of this retrospective study was to evaluate the ultrasonographic (US) features of traumatic neuromas in breast cancer patients after surgery.

\section{Materials and Methods}

\section{Study Population}

This retrospective study was approved by our Institutional Review Board, and the requirement for written informed consent was waived. A search in our pathology database for entries between January 2000 and April 2016 identified seven patients who had been diagnosed with traumatic neuroma in the chest wall. All patients had a history of mastectomy due to breast cancer before being diagnosed with traumatic neuroma. Only the patients whose ultrasonography images were available to review on the picture archiving and communication system (PACS) were included in this study. Six lesions in five patients were included. We reviewed their medical and radiologic records and also analyzed all records regarding ultrasonography and other imaging modalities that were available for review on the PACS.

\section{Imaging Techniques}

The lesions were not imaged via mammography due to the insufficient volume of the remaining tissue. Breast US was performed by a breast radiologist using $5-12 \mathrm{MHz}$ linear-array transducers with one of two high-resolution ultrasound scanners (HDI 5000, Advanced Technology Laboratories, Bothell, WA, USA; iU22 Ultrasound System, Philips Ultrasound, Bothell, WA, USA). All six lesions were evaluated using color Doppler imaging. Four lesions were analyzed using strain elastography with an ultrasound scanner (iU22 Ultrasound System, Philips Ultrasound).

Positron emission tomography-computed tomography (PETCT) was performed to evaluate three lesions in two patients using a combined PET-CT scanner (Discovery ST, GE Healthcare, Milwaukee, WI, USA). All patients fasted for at least 6 hours before undergoing PET-CT. Serum glucose levels were checked to ensure that the level was $<140 \mathrm{mg} / \mathrm{dL}$. Sixty minutes after the intravenous injection of $370 \mathrm{MBq}$ of ${ }^{18} \mathrm{~F}$-2-deoxy-D-glucose, whole-body PETCT was performed. The computed tomography (CT) images were acquired from the skull base to the upper thigh. Immediately after CT acquisition, positron emission tomography (PET) images were obtained with the patient in the same position.

\section{Image Interpretation and Pathology Diagnosis}

The sonograms were reviewed retrospectively by two radiologists who arrived at a consensus. The two readers had not performed the US imaging in the cases in question. They evaluated the traumatic neuromas according to the following criteria enumerated in the Breast Imaging-Reporting and Data System (BI-RADS) lexicon [11]: size, shape, margin, orientation, internal echo pattern, and vascularity on color Doppler US. The elastography images were classified according to the system developed by Itoh et al. [12]: score 1 , even strain for the entire hypoechoic lesion; score 2, strain in most of the hypoechoic lesion with some areas of no strain; score 3, strain at the periphery of the hypoechoic lesion but not at the center of the lesion; score 4, no strain in the entire hypoechoic lesion, but strain in the surrounding area; and score 5, no strain in the entire hypoechoic lesion or in the surrounding area. The final assessment category was also recorded. In addition, the radiologists evaluated whether a tail sign was present, reflecting focal thickening of the nerve; this is a finding known to be associated with neuroma in other parts of the body $[4,13]$. The PET, CT and fused PET-CT scans were viewed on a dedicated workstation for the two patients who underwent that procedure. All six lesions were diagnosed by excisional biopsy.

\section{Results}

Six lesions in five patients for whom US imaging was available to review on the PACS were included in this study. One patient had two masses in the left chest wall, which was the mastectomy site. All patients were asymptomatic, and all lesions were detected incidentally on US. The median age of patients was 44 years (range, 36 to 63 years) and the mean time of diagnosis of traumatic neuroma after mastectomy was 5.6 years (range, 1.9 to 11.1 years).

On US, all lesions presented as a mass. Five neuromas were located in the upper outer quadrant of the chest wall, and one was in the upper inner quadrant. All lesions were located within the pectoralis muscle layer, near the mastectomy scar. The mean size of the masses was $4.8 \mathrm{~mm}$ (range, 3.9 to $5.5 \mathrm{~mm}$ ). Four (66.7\%) presented an oval shape and a circumscribed margin, while two (33.3\%) presented an irregular shape and indistinct margin. They were all hypoechoic, and two showed a non-parallel orientation. Two lesions in one patient showed internal vascularity on color Doppler examinations (Fig. 1). For the four traumatic neuromas evaluated using elastography, the final elastography scores were $1(n=1)$ (Fig. 2), $4(n=2)$, and $5(n=1)$ (Fig. 3). The final assessment categories were BI-RADS 3 ( $n=2$, probably benign), $4 A$ ( $n=2$, low suspicion of malignancy), and $4 B(n=2$, moderate suspicion of malignancy). In addition, the tail sign was detected in only two masses (33.3\%). Table 1 summarizes the US findings of the traumatic neuromas. PET- 
CT scans in two patients revealed no focal uptake areas in their chest wall.

\section{Discussion}

Traumatic neuromas are not true neoplasms; instead, they involve reactive hyperplasia of neuronal and fibrous tissue of the nerve sheath that occurs in the proximal nerve stump after nerve injury [14]. There are two types of traumatic neuromas: spindle neuromas and terminal neuromas. Spindle neuromas occur in a non-severed nerve as a result of chronic irritation. Terminal neuromas occur after the partial or complete transection of a nerve, usually as a result of surgery [15]. The most common locations of terminal neuromas are the lower extremities, followed by the head and neck [2]. They

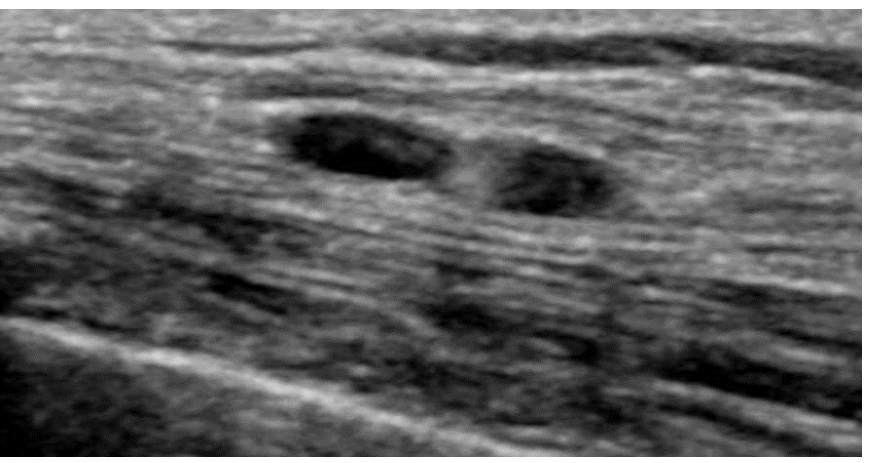

A

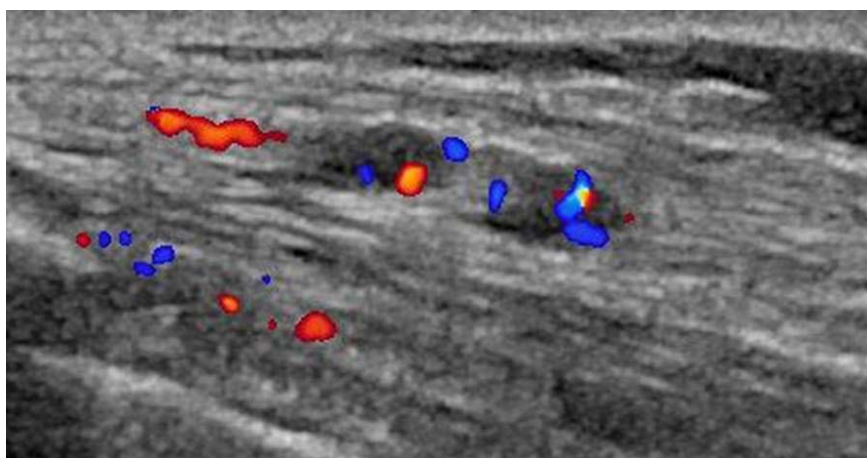

B

Fig. 1. A 36-year-old woman presented with no symptoms.

A. Sonogram shows two oval-shaped masses with circumscribed margins and parallel orientations in the left upper outer chest wall (in the pectoralis major muscle). The patient had undergone left mastectomy for breast cancer 3.2 years previously. B. Color Doppler images show internal vascularity in two masses. The two masses were ultimately diagnosed as traumatic neuromas.

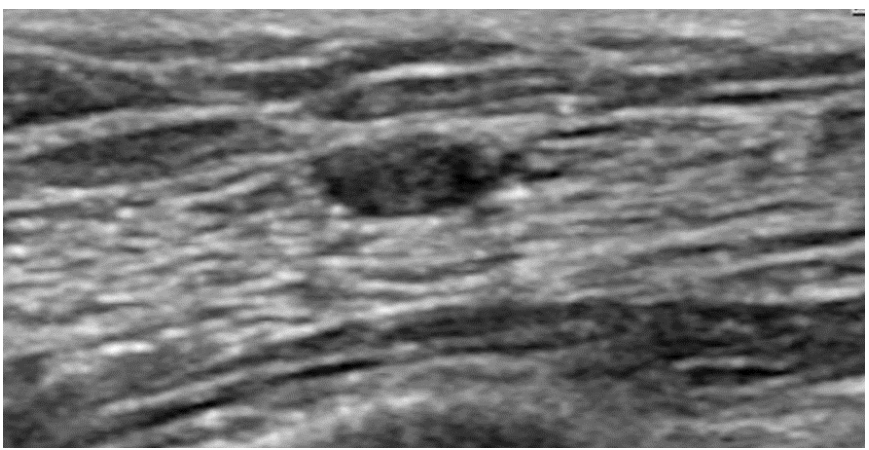

A

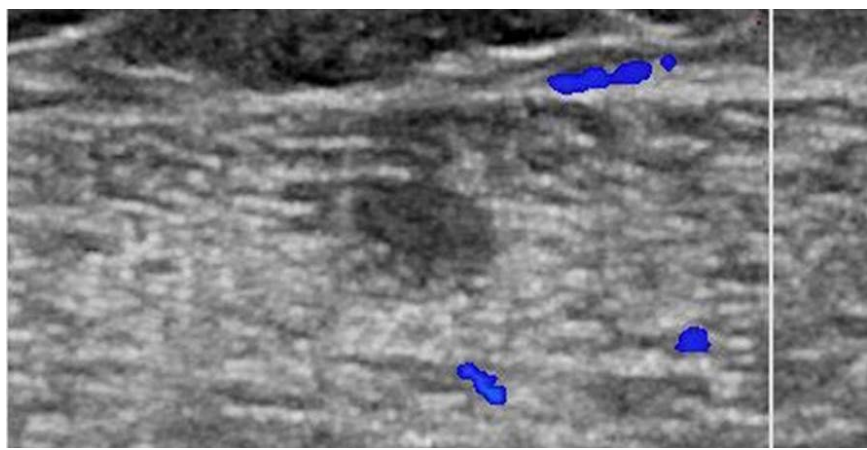

B

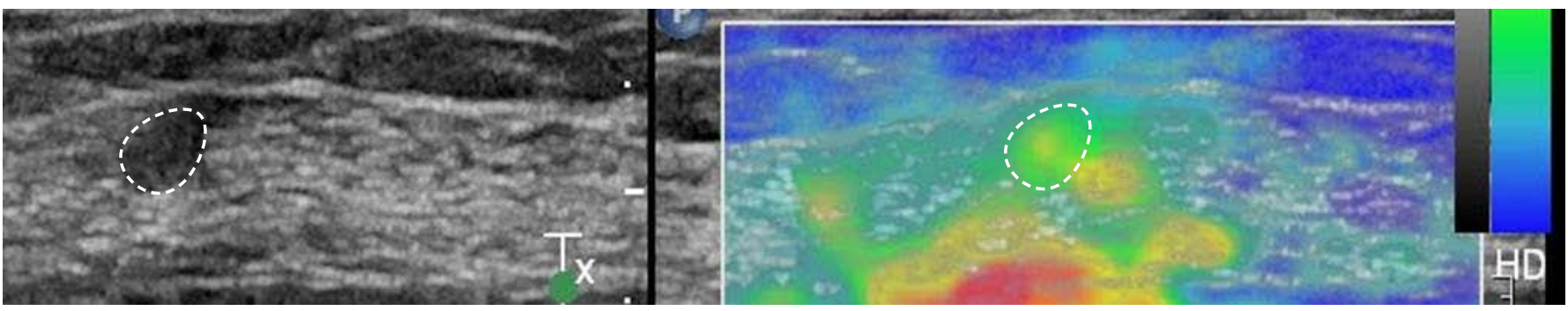

C

Fig. 2. A 63-year-old woman presented with no symptoms.

A. Sonogram shows an oval-shaped mass with a circumscribed margin and parallel orientation in the left upper outer chest wall (in the pectoralis major muscle). The patient had undergone left mastectomy for breast cancer 10 years previously. B. A color Doppler image shows no internal vascularity. C. Strain elastography shows an elasticity score of 1. 


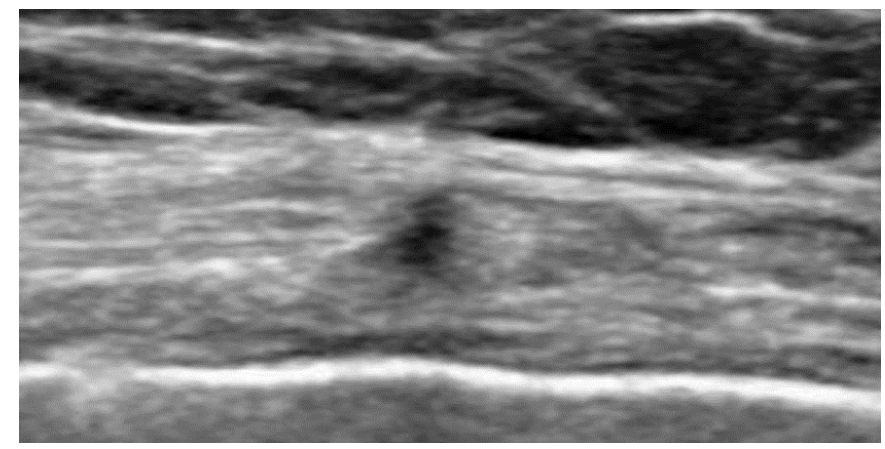

A

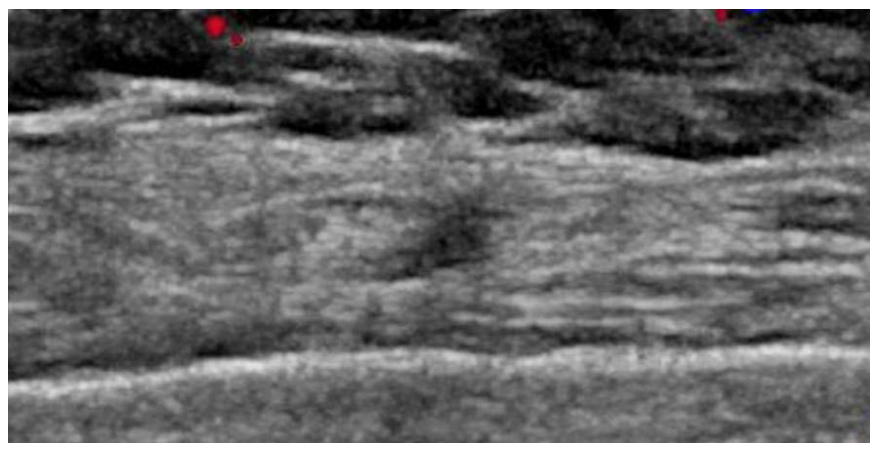

B

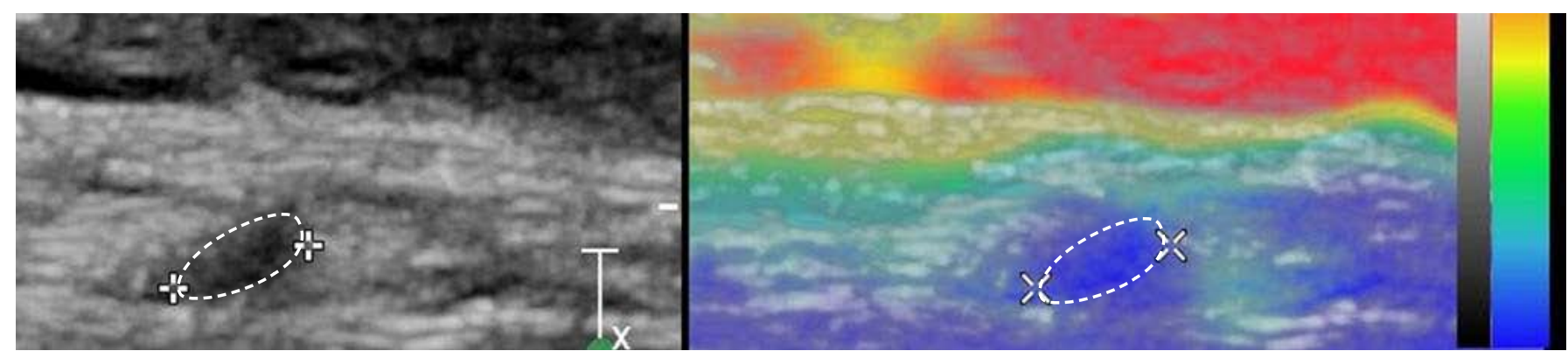

C

Fig. 3. A 62-year-old woman presented with no symptoms.

A. Sonogram shows an irregularly shaped mass with an indistinct margin and non-parallel orientation in the right upper outer chest wall (in the pectoralis major muscle). The patient had undergone right mastectomy for breast cancer 11.1 years previously. B. A color Doppler image shows no internal vascularity. C. Strain elastography shows an elasticity score of 5.

Table 1. Summary of the sonographic findings of traumatic neuromas according to the BI-RADS lexicon

\begin{tabular}{|c|c|c|c|c|c|c|c|c|c|}
\hline Case No. & Shape & Margin & Orientation & Echo pattern & Color Doppler & Elastography score ${ }^{a)}$ & Tail sign ${ }^{\text {b) }}$ & Location & BI-RADS \\
\hline 1 & Oval & Circumscribed & Parallel & Hypo & $\mathrm{N} / \mathrm{A}$ & N/A & - & L upper inner & C3 \\
\hline 2 & Irregular & Indistinct & Non-parallel & Нypo & N/A & N/A & + & L upper outer & C4B \\
\hline 3 & Oval & Circumscribed & Parallel & Hypo & + & 4 & - & L upper outer & $\mathrm{C} 4 \mathrm{~A}$ \\
\hline 4 & Oval & Circumscribed & Parallel & Hypo & + & 4 & - & L upper outer & $\mathrm{C} 4 \mathrm{~A}$ \\
\hline 5 & Oval & Circumscribed & Parallel & Hypo & - & 1 & - & L upper outer & C3 \\
\hline 6 & Irregular & Indistinct & Non-parallel & Нyро & - & 5 & + & R upper outer & $\mathrm{C} 4 \mathrm{~B}$ \\
\hline
\end{tabular}

BI-RADS, Breast Imaging-Reporting and Data System; N/A, not available.

${ }^{\text {a) }}$ Elastography images were classified according to the system developed by Itoh et al. [12]: score 1, even strain for the entire lesion; score 2, strain in most of the lesion with some areas of no strain; score 3, strain at the periphery of the lesion but not in the center; score 4, no strain in the entire lesion; and score 5, no strain in the entire lesion and

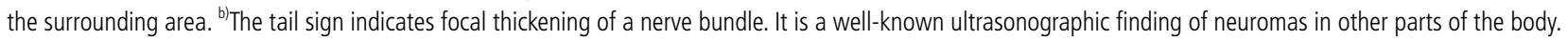

very rarely occur in the breast or chest wall after breast cancer surgery. Alsharif et al. [4] reported that the incidence of traumatic neuromas in the breast was $0.09 \%$. According to previous reports, traumatic neuromas most commonly present on US as oval-shaped, hypoechoic masses with a circumscribed margin and no internal vascularity on color Doppler examinations. In our study, most traumatic neuromas likewise presented as oval-shaped, hypoechoic masses with a circumscribed margin $(n=4,67 \%)$. However, unlike previous reports, two traumatic neuromas showed internal vascularity on color Doppler examinations in our study. Only one study has assessed traumatic neuromas using strain elastography [4]. In that study, three neuromas (in two patients) showed a benign elasticity score of 1 or 2 . However, in our study, two masses showed an elasticity score of 4 and one mass showed an elasticity score of 5. Therefore, in contrast to previous reports [4], traumatic neuromas occurring in the chest wall of breast cancer patients after 
mastectomy can show increased vascularity on color Doppler images and may present as a hard mass on elastography.

Previously reported traumatic neuromas after mastectomy usually occurred near the surgical site, most commonly in the lateral aspect of chest wall due to the high incidence of malignancies in this location [2-10]. In our study, five of the six traumatic neuromas were located in the upper outer aspect of the chest wall, and one was in the upper inner aspect. All six lesions were located near the surgical scar. This finding is likely related to the postsurgical damage of the nerves that innervate the breast. During mastectomy, branches of the long thoracic, thoracodorsal, lateral pectoral, and medial pectoral nerves, as well as cutaneous branches of the intercostal nerves, are susceptible to injury [16]. Anatomically, the medial and lateral pectoral nerves and cutaneous branches of the fourth through sixth intercostal nerves are located close to the pectoralis major muscle [2]. Furthermore, some branches pass through the pectoralis major muscle. Thus, these nerves may be compromised during division of the pectoralis muscle [17]. In our study, all lesions were found in the pectoralis muscle layer. This may be helpful in differentiating traumatic neuromas from cases of local recurrence, as most local recurrences after mastectomy tend to be found in the subcutaneous fat layer of the chest wall.

The local recurrence rates in the chest wall after mastectomy have been reported to range from $5 \%$ to $27 \%$, and approximately $80 \%$ of recurrences take place within the first 5 years after surgery [18]. AlSharif et al. [4] reported that the mean time between mastectomy and the detection of a neuroma was 10.3 years (range, 2 to 22 years), and Wang et al. [6] reported that three of six neuromas were detected more than 5 years after mastectomy. However, the mean time to diagnosis of the traumatic neuromas after mastectomy in our study was 5.6 years (range, 1.9 to 11.1 years). Four of the six traumatic neuromas were detected within the first 5 years after mastectomy. Patients who undergo mastectomy for breast cancer usually undergo surveillance US in Korea. This may explain why traumatic neuromas in this study were detected earlier than in previous reports.

Our study had several limitations. First, the number of enrolled patients was small, and we therefore could not obtain statistically significant results. Second, the retrospective nature of the study most likely limited our results. Only lesions that were histologically confirmed as neuromas were included in this study, and some selection bias was unavoidable. The retrospective evaluation of images prevented us from analyzing US findings in real time, which might have influenced the analysis performed by the readers. Third, since insufficient previous reports have been published, the diagnostic value of elastography for chest wall lesions remains unclear.
In conclusion, an oval shape, circumscribed margin, parallel orientation, and hypoechogenicity appear to be the most frequent US features of traumatic neuromas in breast cancer patients after mastectomy. Neuromas may show increased vascularity on color Doppler imaging and present as a hard mass on elastography.

ORCID: Hwa Sung Sung: http://orcid.org/0000-0003-4646-9528; Young-Seon Kim: http://orcid.org/0000-0002-9168-8204

\section{Conflict of Interest}

No potential conflict of interest relevant to this article was reported.

\section{References}

1. Foltan R, Klima K, Spackova J, Sedy J. Mechanism of traumatic neuroma development. Med Hypotheses 2008;71:572-576.

2. Kim EY, Kang DK, Kim TH, Kim KS, Yim H. Traumatic neuroma in a breast cancer patient after modified radical mastectomy: a case report. J Korean Soc Radiol 2011;64:515-518.

3. Ducic I, Seiboth LA, lorio ML. Chronic postoperative breast pain: danger zones for nerve injuries. Plast Reconstr Surg 2011;127:41. 46.

4. AlSharif S, Ferre R, Omeroglu A, El Khoury M, Mesurolle B. Imaging features associated with posttraumatic breast neuromas. AJR Am J Roentgenol 2016;206:660-665.

5. Shin S. A traumatic neuroma in breast cancer patient after mastectomy: a case report. J Korean Soc Radiol 2013;69:405-407.

6. Wang $X$, Cao $X$, Ning L. Traumatic neuromas after mastectomy. ANZ J Surg 2007;77:704-705.

7. Rosso R, Scelsi M, Carnevali L. Granular cell traumatic neuroma: a lesion occurring in mastectomy scars. Arch Pathol Lab Med 2000;124:709-711.

8. Ashkar L, Omeroglu A, Halwani F, Alsharif S, Loutfi A, Mesurolle B. Post-traumatic neuroma following breast surgery. Breast J 2013;19:671-672.

9. Li Q, Gao EL, Yang YL, Hu HY, Hu XQ. Traumatic neuroma in a patient with breast cancer after mastectomy: a case report and review of the literature. World I Surg Oncol 2012;10:35.

10. Baltalarli B, Demirkan N, Yagci B. Traumatic neuroma: unusual benign lesion occurring in the mastectomy scar. Clin Oncol (R Coll Radiol) 2004;16:503-504.

11. Mendelson EB, Bohm-Velez M, Berg WA, Whitman GJ, Feldman MI, Madjar $\mathrm{H}$, et al. ACR BI-RADS ultrasound. In: D'Orsi CJ, Sickles EA Mendelson EB, Morris EA, eds. ACR BI-RADS Atlas, Breast Imaging Reporting and Data System. 5th ed. Reston, VA: American College of Radiology, 2013;1-173.

12. Itoh A, Ueno E, Tohno E, Kamma H, Takahashi H, Shiina T, et al Breast disease: clinical application of US elastography for diagnosis. Radiology 2006;239:341-350. 
13. Abe S, Nakao T, Yamane S, Fukuda M, Yamamoto M, Santti R, et al. Morphology of plantar interdigital neuroma: a comparative cadaveric study of elderly Finnish and Japanese individuals. Okajimas Folia Anat Jpn 2013;90:1-5.

14. Yabuuchi H, Kuroiwa T, Fukuya T, Tomita K, Hachitanda Y. Traumatic neuroma and recurrent lymphadenopathy after neck dissection: comparison of radiologic features. Radiology 2004;233:523-529.

15. Boutin RD, Pathria MN, Resnick D. Disorders in the stumps of amputee patients: MR imaging. AJR Am J Roentgenol 1998;171:497501.
16. Wong L. Intercostal neuromas: a treatable cause of postoperative breast surgery pain. Ann Plast Surg 2001;46:481-484.

17. Moosman DA. Anatomy of the pectoral nerves and their preservation in modified mastectomy. Am J Surg 1980;139:883886.

18. Yilmaz MH, Esen $G$, Ayarcan $Y$, Aydogan F, Ozguroglu M, Demir $G$, et al. The role of US and MR imaging in detecting local chest wall tumor recurrence after mastectomy. Diagn Interv Radiol 2007;13:13-18. 